\title{
Erstmanifestation eines systemischen Lupus \\ P. Seele \\ H.-D. Göring erythematodes unter dem Bild flagellatenförmiger Erytheme in der Glutealregion
}

\author{
Flagellate Erythema at the Buttock as an Initially Manifestation of a Systemic \\ Lupus Erythematodes
}

\section{Zusammenfassung}

Wir berichten über eine 51-jährige Patientin mit einem systemischen Lupus erythematodes (SLE), der initial allein durch ungewöhnliche und bisher in der Literatur für SLE noch nicht beschriebene flagellatenförmige Erytheme in der Gesäßregion auffällig wurde. Diese Hautveränderungen allein ließen an flagellatenförmige Erytheme denken, wie sie bei Dermatomyositis, als Folge einer Bleomycin-Therapie und bei der so genannten Shiitake-Dermatitis beschrieben wurden. Durch unsere anamnestischen Erhebungen, klinischen, histologischen und immunologischen Untersuchungen konnten wir einen SLE bestätigen und die anderen Erkrankungen ausschließen. Unter einer Therapie mit Prednisolon, Azathioprin und Chloroquin heilten die flagellatenförmigen Erytheme bei unserer Patientin ab. Die Ätiologie und Pathogenese der flagellatenförmigen Erytheme bleiben unklar. In unserem Fall spricht vieles dafür, dass es sich bei den flagellatenförmigen Erythemen um eine Manifestation des SLE handelt, dessen besondere Morphologie durch Kratzen bei Pruritus geprägt wurde.

\section{Abstract}

First report in the literature on a 51-year-old woman with a systemic lupus erythematodes (SLE), which initially appeared as flagellate erythema on the buttock resembling suchlike described in dermatomyositis, after bleomycin therapy and in the so called shiitake dermatitis. By means of our anamnestic, clinical, histological and immunological investigations we could confirm the diagnosis SLE and exclude the other diseases and possibilities. The flagellate erythema in our patient resolved completely under treatment with prednisolone, azathioprine and chloroquine. Etiology and pathogenesis of the flagellate erythema remain unclear. In our case we suppose that the flagellate erythema is a skin manifestation of the SLE, triggered by scratching.

\section{Einleitung}

Die Hauterscheinungen eines Lupus erythematodes (LE) können äußerst vielgestaltig sein. Das initiale Auftreten von seltenen und ungewöhnlichen Hautveränderungen (Tab.1) ohne sonstige klinische Beschwerden kann die Diagnosestellung und damit auch eine Therapieeinleitung verzögern. Wir beobachteten erstmals eine Patientin, die bei der Vorstellung in unserer Klinik zunächst als einziges Symptom eines SLE flagellatenförmige Erytheme aufwies. Im Zuge der anschließenden Diagnostik konnte ein SLE gesichert und erfolgreich behandelt werden. Der Kasus unterstreicht die Bedeutung der Kenntnis von Hautveränderungen für die Diagnose des SLE und die Abgrenzung von anderen Krankheitsbildern, die mit ähnlichen Hauterscheinungen einhergehen, und soll daher hier berichtet werden. 
Tab. 1 Kutane Manifestationen des Lupus erythematodes (aus [6])

systemischer Lupus erythematodes (SLE)
bullöser SLE (BLE)
Urtikariavaskulitis
subakut kutaner LE (SCLE)
diskoider Lupus erythematodes (DLE)
Lupus erythematodes tumidus (LET)
Lupus erythematodes hypertrophicus/verrucosus (LEHV)
Chilblain Lupus erythematodes
Lupus erythematodes profundus
seltene kutane Manifestationen des Lupus erythematodes
anuläres Erythem
papulöse Muzinose

\section{Kasuistik}

\section{Anamnese}

Im Januar 2000 traten bei der damals 51-jährigen Patientin in ihrer Entstehung unklare, multiple, gering juckende, streifenförmige Hautveränderungen am Gesäß auf, welche als Casus pro diagnosi zur Vorstellung in unserer Klinik führten. Auf genauere Befragung gab die Patientin an, dass einige Monate vorher 2 flache oberflächliche fünfmarkstückgroße Ulzera im Glutealbereich bestanden hatten, die nach lokaler Steroidanwendung abgeheilt waren. Schließlich berichtet sie über Schmerzen in den Schultern, Knien, Hand- und Fingergelenken, die seit mehreren Jahren bestanden und deren Ursache nicht geklärt war.

\section{Aufnahmebefund}

Im Bereich der Glutealregion und der angrenzenden Oberschenkelbeugeseiten finden sich beidseits längsgestellte, bis $8 \mathrm{~cm}$ lange und $3 \mathrm{~cm}$ breite, erythematös-livide, flagellatenförmige, scharf begrenzte und gering infiltrierte Hautveränderungen (Abb.1).

Bis auf einzelne Exkoriationen über dem Os sacrum war das übrige Integument hauterscheinungsfrei. Hautveränderungen und Gelenkbeschwerden ließen uns an eine Kollagenose denken. Wir leiteten daraufhin eine ausgedehnte klinische, laborchemische immunologische und apparative Diagnostik ein.

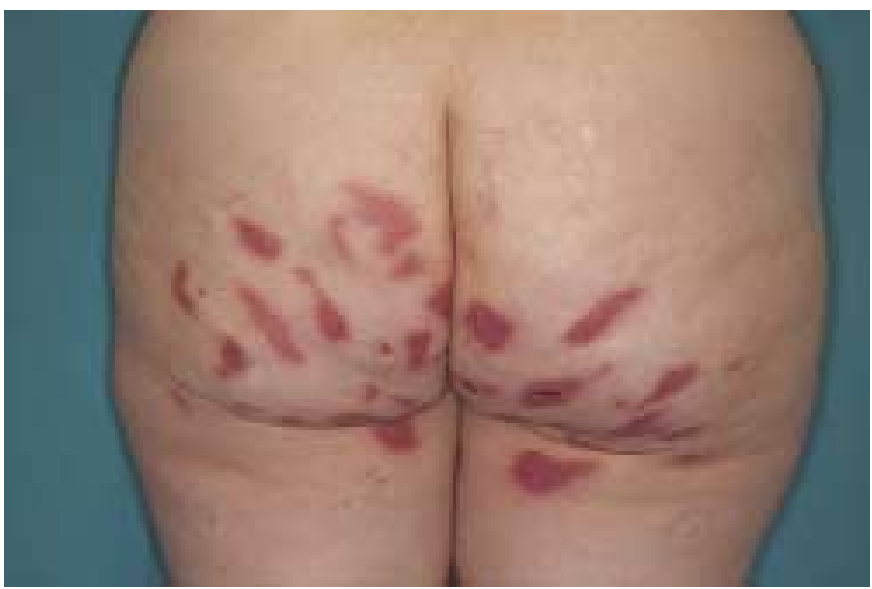

Abb. 1 Flagellatenförmige Erytheme der Glutealregion.

\section{Befunde diagnostischer Untersuchungen Klinische Chemie}

BSG: mit 20/32 mm mäßig beschleunigt; alkalische Phosphatase: 5,25 (NW < 4,0); 24-h-Sammelurin auf Eiweiß: 0,15 g (grenzwertig pathologisch); Blutzucker: max. 7,2 mmol/l. Blutbild, Kreatinin, Kreatininclearance, Elektrolyte, Harnstoff, Harnsäure, ALAT, ASAT, gGT, CRP, CK, LDH, Gerinnung und RF im Normbereich.

\section{Immunologische Befunde}

ANF: 1 : 1280 (trivalent, homogen; Substrat: Hep2-Zellen) dsDNS-AK: $1: 2560$ (Substrat: Crithidia luciliae) Phospholipid-AK-Screen: Cardiolipin-AK-Screen 51U/ml (NW: < 45), B2-Glycoprotein-I-AK-Screen: positiv

ENA-Screen, AMAK, p-/c-ANCA, ribosomale AK, AK gegen glatte und quergestreifte Muskulatur, M2-AK: nicht nachweisbar; ZIK, Immunglobuline, C3 und C4 im Serum im Normbereich; kein Paraprotein im Serum und Urin

\section{Histologie (läsionale Haut)}

Haut mit Anhangsgebilden. Orthohyperkeratose mit teilweise Serokrusten-Einlagerungen. Akanthose der Epidermis. Ödem im Stratum papillare. Überwiegend im oberen Korium vermehrte und z.T. wandverdickte Kapillaren. Superfizielle und tiefe perivaskuläre lymphozytäre Infiltrate, teilweise auch perifollikuläre lymphozytäre Infiltrate. In der Alzianblaufärbung geringe $\mathrm{Mu}$ zinablagerungen subepidermal (Abb. 2).

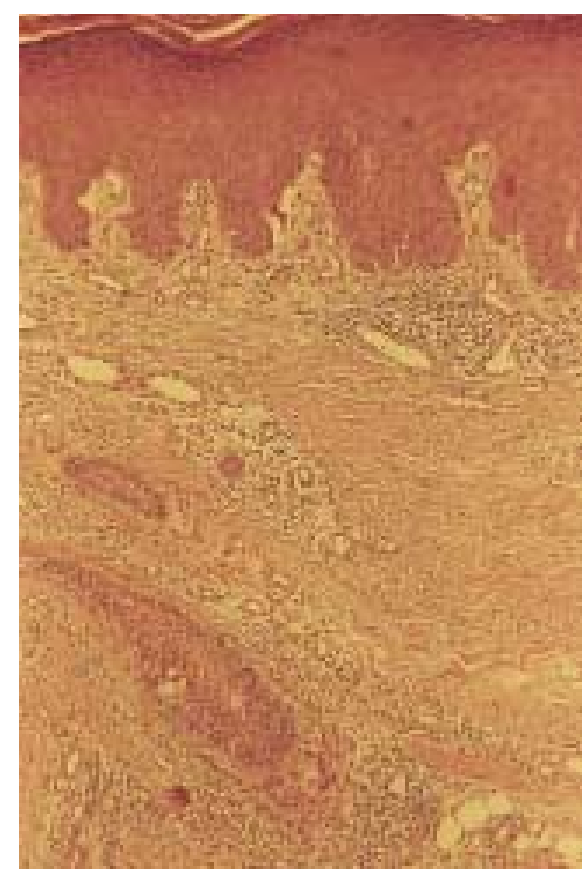

Abb. 2 Histologie der flagellatenförmigen Erytheme mit perivaskulären lymphozytären Infiltraten im Korium und epidermalen Veränderungen durch Kratzeffekte. Färbung: H.E.; Vergrößerung: $25 \times$

\section{DIF (läsionale Haut, Gesäß)}

bandförmige IgG- und C3-Niederschläge entlang der dermoepidermalen Junktionszone

\section{DIF (unbefallene Haut, Oberarminnenseite)}

bandförmige IgG- und C3-Niederschläge entlang der dermoepidermalen Junktionszone: positiver Lupusband-Test 


\section{Apparative Untersuchungen und fachärztliche Konsile}

Röntgen-Thorax: Verdacht auf kleinen Pleuraerguss links; laterale Sinusadhärenzen; Aortenelongation und -sklerose; sonst regelrecht

Pleurasonographie: kein Anhalt für frischen Erguss, am ehesten laterale Zwerchfelladhäsionen

Echokardiographie: regelrechter Befund, insbesondere kein Anhalt für Perikarderguss

Ergo-Spirometrie: regelrechter Befund

Sonographie Abdomen und Nieren: regelrechter Befund

Speicheldrüsenszintigraphie: Normalbefund

Ösophagus-Breischluck-Passage: regelrechter Befund

Ösophagusszintigraphie: zeitlich normale Passage, jedoch auffällige Pendelbewegung, die als beginnende Funktionsstörung gewertet werden könnte

orthopädisches Konsil: Arthralgien bei SLE

neurologisches Konsil: kein Anhalt für Myositis

\section{Therapie und Verlauf}

Wir therapierten initial mit $80 \mathrm{mg}$ Prednisolon, $100 \mathrm{mg}$ Imurek und $250 \mathrm{mg}$ Resochin pro Tag. Darunter heilten bereits unter stationären Bedingungen die Hauterscheinungen vollständig ab. Im weiteren ambulanten Verlauf konnten wir die Prednisolondosis auf eine Erhaltungsdosis von jetzt $5 \mathrm{mg}$ senken, die Imurekdosis reduzierten wir auf $50 \mathrm{mg}$ pro Tag. Darunter sind bis zum heutigen Tag keine neuen Hauterscheinungen aufgetreten, auch die Gelenkbeschwerden haben sich deutlich gebessert und die Patientin fühlt sich wohl.

\section{Diskussion}

Die Patientin stellte sich primär ausschließlich wegen der ungeklärten auffälligen flagellatenförmigen Erytheme in unserer Klinik vor. Prima vista ließen uns die hier beschriebenen Hautveränderungen am Gesäß wegen ihrer Lokalisation, Form, Anordnung und Farbe an relativ frische Verletzungen durch Misshandlungen (Schläge) denken. Dafür gab es jedoch anamnestisch keine Bestätigung. Auf gezieltes Befragen konnten zusätzlich relativ diskrete Gelenkbeschwerden eruiert werden, die allein nicht wegweisend für das Vorliegen eines SLE waren. Ausschließlich die Hauterscheinungen ließen uns u.a. auch an einen LE denken und waren Veranlassung für eine entsprechende Labordiagnostik. Nachdem der klinische LE-Verdacht durch die immunologischen und histologischen Befunde bestätigt wurde, konnten durch nochmalige subtile Erhebung der Anamnese und Gewinnung von klinischen sowie paraklinischen Daten weitere ARAKriterien und sichere sowie mögliche Hinweise auf eine interne Manifestation eines SLE gewonnen werden. Folgende ARA-Kriterien waren erfüllt: hohe ANA-Titer, Nachweis von ds-DNS-AK, Hauterscheinungen eines LE, sonographisch und röntgenologisch Pleuraadhäsionen (im Sinne eines Zustandes nach Pleuraergüssen beidseits) und Arthralgien.

Der vorgestellte Kasus ist ein Beispiel für die Kompetenz des Dermatologen auf dem Gebiet der Kollagenosen. Die richtige Bewertung der als einziges augenfälliges Symptom bestehenden Hautveränderungen führte zur Diagnose.
In einer Übersichtsarbeit [6] wurde über seltene kutane Manifestationen des Lupus erythematodes berichtet, welche durchaus diagnostische Schwierigkeiten bereiten können. Die von uns beobachteten flagellatenförmigen Hauterscheinungen sind in dieser Arbeit nicht beschrieben. Auch in ausgedehnten Literaturrecherchen konnten flagellatenförmige Erytheme bei SLE-Patienten nicht gefunden werden, so dass es sich bei unserem Fall um die erste Mitteilung handelt.

Über das Auftreten flagellatenförmiger Erytheme wird bei einigen Fällen von Dermatomyositis $[4,5,8,9]$, nach Bleomycin-Therapie $[1,2]$ und nach dem Genuss des ungekochten Speisepilzes Lentinus edodes bei der so genannten Shiitake-Dermatitis [3,7] berichtet.

Bei unserer SLE-Patientin konnte eine Dermatomyositis zweifelsfrei ausgeschlossen werden. Eine Bleomycin-Therapie und der Genuss von Speisepilzen schieden bei ihr anamnestisch aus. Sie ist damit der erste publizierte Kasus eines SLE mit flagellatenförmigen Erythemen. Das ist auch insofern bemerkenswert, als bisher die Ansicht galt, dass flagellatenförmige Erytheme innerhalb der Kollagenosen ausschließlich bei Dermatomyositis auftreten $[4,8]$. Die Einzelmorphe der Hauterscheinungen bei unserer SLE-Patientin entsprach morphologisch den flagellatenförmigen Erythemen, wie sie bei Dermatomyositis, nach BleomycinTherapie und bei Shiitake-Dermatitis beschrieben werden. Topographisch lag bei unserer Patientin mit dem Befall der Gesäßregion und der angrenzenden Oberschenkelbeugeseiten eine bevorzugte Lokalisation des Rumpfes vor, wie sie auch bei flagellatenförmigen Erythemen in Verbindung mit Dermatomyositis, Bleomycin-Therapie und Shiitake-Dermatitis beschrieben wird [1-9].

Zusätzlicher Befall von Extremitäten, Kopf und Nacken ist möglich. Ein mehr oder weniger ausgeprägter Pruritus, wie ihn unsere Patientin schilderte, wird auch bei flagellatenförmigen Erythemen im Zusammenhang mit den anderen Krankheiten und Störungen geschildert [1-9]. Bei Bleomycin-induzierten flagellatenförmigen Erythemen scheint eine etwas deutlichere Neigung zu postinflammatorischer Hyperpigmentierung zu bestehen $[1,2]$. Bei der weitgehenden klinischen Übereinstimmung der flagellatenförmigen Erytheme bei unterschiedlichen Grundleiden stellt sich die Frage nach histologischen Veränderungen und eventuellen ätiopathogenetischen Gemeinsamkeiten. Die Erytheme bei unserer Patientin wiesen histologische Veränderungen auf, wie sie auch bei einem SLE auftreten können. Die beschriebenen epidermalen Befunde können Ausdruck von Kratzeffekten sein (Abb.2).

In der Epidermis können v.a. bei Dermatomyositis-assoziierten flagellatenförmigen Erythemen eine Hyperkeratose mit fokaler Parakeratose und unregelmäßiger Akanthose, Spongiose und Exozytose von Lymphozyten auftreten [1,5,9]. Die histologischen Veränderungen der Bleomycin-induzierten flagellatenförmigen Erytheme zeigen eine weitgehende Übereinstimmung mit denen der Interface-Dermatitis bei Kollagenosen [1]. In 5 Tage alten Bleomycin-induzierten flagellatenförmigen Erythemen fand sich in einem Fall im Biopsiematerial eine lymphozytäre Vaskulitis [2]. Bei Shiitake-Dermatitis konnten in den flagellatenförmigen Erythemen eine Spongiose und nekrotische Zellen der Epi- 
dermis und ein Ödem sowie perivaskuläre lymphohistiozytäre Infiltrate in der Dermis nachgewiesen werden [3].

Nur wenige Angaben liegen über die Ergebnisse der direkten Immunfluoreszenzuntersuchung (DIF) bei flagellatenförmigen Erythemen vor. Es fanden sich granuläre Niederschläge von IgM und C3 in der Basalmembranzone bei Dermatomyositis-assoziierten flagellatenförmigen Erythemen [5], andere Autoren berichten über negative DIF-Befunde [4,9]. Bei Bleomycin-induzierten flagellatenförmigen Erythemen zeigten sich in der DIF C3- und Fibrinogen-Niederschläge in den Gefäßwänden der mittleren und tieferen Dermis [2]. Die bei unserer SLE-Patientin in den flagellatenförmigen Erythemen nachgewiesenen IgG- und C3-Ablagerungen in der dermoepidermalen Junktionszone und der positive Lupusband-Test entsprechen den zu erwartenden Veränderungen des SLE und sind keine flagellatenförmige Erytheme-typischen Befunde.

Ätiologie und Pathogenese der flagellatenförmigen Erytheme sind bis heute nicht geklärt. Es fällt auch schwer, diesbezüglich Gemeinsamkeiten der flagellatenförmigen Erytheme bei unterschiedlichen Grundleiden und Störungen zu erkennen.

In der Ätiopathogenese der flagellatenförmigen Erytheme bei Dermatomyositis wird neben mechanischen Traumen, die bis zur Auslösung eines Koebner-Phänomens reichen können, eine Sonnenlichtexposition diskutiert $[1,2,4,5,8]$. Zusätzlich könnte eine Myositis zur Zerstörung der glatten Muskulatur kleiner Gefäße und damit zu einer daraus resultierenden Vasodilatation führen [9]. Mechanische Reize in Form von Kratzeffekten, ausgelöst durch den Pruritus, werden im Falle der Bleomycin-induzierten flagellatenförmigen Erytheme für möglich gehalten. Die dadurch hervorgerufene Gefäßerweiterung führt zu einer lokalen Anreicherung und toxischen Wirkung von Bleomycin [2].

Toxische Effekte, eine Lichtsensibilisierung und eine Freisetzung von gefäßerweiternden Zytokinen durch das in Lentinus edodes enthaltene Polysaccharid Lentinan werden für die Entstehung der flagellatenförmigen Erytheme der Shiitake-Dermatitis verantwortlich gemacht. Das entzündliche Zellinfiltrat wird in diesem Zusammenhang als Antwort auf einen Antigenreiz interpretiert [3]. Nach den klinischen, histologischen und immunologi- schen Befunden handelt es sich bei den flagellatenförmigen Erythemen unserer SLE-Patientin in erster Linie um Hauterscheinungen eines SLE. Ihre charakteristische Ausprägung könnte u.a. durch Kratzen wegen eines geklagten Pruritus mitbedingt sein. Die flagellatenförmigen Erytheme unserer SLE-Patientin heilten relativ schnell unter einer immunsuppressiven Therapie mit Prednisolon, Azathioprin und Chloroquin ab. Der zusätzliche Einsatz von Chloroquin (Resochin ${ }^{\circledR}$ ) bei SLE-Patienten mit Hautund/oder Gelenkbeteiligungen hat sich bei uns seit vielen Jahren bewährt. Bei Dermatomyositis wird ebenfalls die Rückbildung der assoziierten flagellatenförmigen Erytheme unter Prednisolon und Azathioprin [5], Prednisolon-Monotherapie [9], und sogar spontan $[4,9]$ beschrieben. Bleomycin-induzierte flagellatenförmige Erytheme klangen nach Absetzen der Bleomycin-Therapie und Gabe von Antihistaminika [2] und Kombination von Glukokortikoiden und Antihistaminika [1] ab. Die flagellatenförmigen Erytheme der Shiitake-Dermatitis heilten nach Verzicht auf den Pilzgenuss und oraler Gabe von Antihistaminika sowie lokaler Anwendung von steroidhaltigen Externa ab [3].

\section{Literatur}

${ }^{1}$ Cortina P, Garrido JA, Tomas JF, Unamuno P, Armino M. "Flagellate" erythema from bleomycin. With histopathological findings suggestive of inflammatory oncotaxis. Dermatologica 1990; 180: 106-109

2 Duhra P, Ilchyshyn A, Das RN. Bleomycin-induced flagellate erythema. Clin Exp Dermatol 1991; 16: 216-217

${ }^{3}$ Hanada K, Hashimoto I. Flagellate mushroom (Shiitake) dermatitis and photosensitivity. Dermatology 1998; 197: 255-257

${ }^{4}$ Jara M, Amerigo J, Duce S, Borbujo J. Dermatomyositis and flagellate erythema. Clin Exp Dermatol 1996; 21: 440-441

${ }^{5}$ Kimyai-Asadi A, Tausk FA, Nousari HC. A patient with dermatomyositis and linear streaks on the back. Centripetal flagellate erythema (CFE) associated with dermatomyositis. Arch Dermatol 2000; 136: 667-670

${ }^{6}$ Kuhn A, Schuppe H-C, Rusicka T, Lehmann P. Seltene kutane Manifestationen des Lupus erythematodes. Hautarzt 2000; 51: 818-825

${ }^{7}$ Nakamura T. Toxicoderma caused by shiitake (Lentinus edodes) (in Japanese). Jap J Clin Dermatol 1977; 31: 65-68

${ }^{8}$ Nousari HC, Ha VT, Laman SD, Provost TT, Tausk FA. "Centripetal flagellate erythema". A cutaneous manifestation associated with dermatomyositis. J Rheumatol 1999; 26: $692-695$

${ }^{9}$ Watanabe T, Tsuchida T. "Flagellate" erythema in dermatomyositis. Dermatology 1995; 190: 230-231 